EPJ Web of Conferences 71, 00078 (2014)

DOI: $10.1051 /$ epjconf/20147100078

(C) Owned by the authors, published by EDP Sciences, 2014

\title{
Undulator Spin Polarimetry for the "precursor" storage ring eEDM experiment
}

\author{
Alfredo U. Luccio \\ ${ }^{1}$ guest, Brookhaven National Laboratory, Upton, New York e-mail: luccio@bnl.gov
}

\begin{abstract}
In a precursor eEDM experiment to the proton EDM, spin polarimetry based on undulator radiation is proposed.
\end{abstract}

\section{The EDM storage ring experiment(s)}

We plan a storage ring based experiment (100 M\$) to measure with an accuracy of $10^{-29} \mathrm{e}-\mathrm{cm}$ the electric dipole moment (EDM) of the proton. In a second "precursor" storage ring experiment we propose to measure the electric dipole moment of the electron. The eEDM ring will be smaller and much less expensive than the pEDM ring and might be constructed first.

The e-ring will also be useful as a model to test the not-so-well known accelerator physics of an electric storage ring.

In the pEDM we will use polarized protons at the "magic" (see next) momentum of $0.7 \mathrm{Gev} / \mathrm{c}$. In the precursor eEDM, polarized electrons at the "magic" momentum of $15 \mathrm{MeV} / \mathrm{c}$. The EDM will be measured by spin polarimetry.

The rings will be strictly electrostatic (no magnets). At the magic momentum, in absence of magnetic fields, the spin direction will remain "frozen" in its direction at injection (longitudinal) and the EDM will be measured as proportional to a small vertical component of the spin that will gradually appear. Interested people may check another parallel talk to this Conference[1].

\subsection{Synchrotron radiation and spin polarimetry}

It was suggested[2] that synchrotron radiation by polarized particles in an accelerator will show an extra tail. This effect was finally measured on electrons [3]. SR is due to the radiation by an accelerated charged particle (in particular, oscillating.) The tail is due to the radiation of an oscillating momentum. It is a second order radiation field. This component of SR goes with the inverse square of the mass of the particle, so it is much weaker for protons than for electrons.

It seems already promising to do spin polarimetry by ordinary wide-spectrum SR, however there are better news. If one enhances synchrotron radiation using an undulator in the ring (say electrostatic to preserve the magic condition), SR becomes more intense, more coherent and, very important, shows a line spectrum. It turns out that the monopole and the dipole spectral lines have opposite polarization.

\footnotetext{
${ }^{1}$ We acknowledge the support of the U.S. Department of Energy
} 
So, a spectrometer sensitive to light polarization will serve as a spin polarimetry. We present some practical numbers in the following.

Actually, electron spin polarimetry was done at CERN-LEP by laser scattering, which works on the same physical principles, because an undulator is seen in the frame of an incoming particle as an EM wave that paradoxically propagate, not at the speed of light, $c$, but at the speed of the particle $\beta c$. However, laser polarimetry is not practical in the case at hand.

\subsection{The magic condition}

Spin dynamics is governed by the covariant T-BMT equation

$$
\frac{d \mathbf{s}}{d t}=-\frac{q}{m \gamma} \mathbf{f} \times \mathbf{s}
$$

where $\mathbf{s}$ is a real 3-dimensional spin vector of a 1/2-spin particle, and $\mathbf{f}$ is a function of the position and the momentum of the particle and of the electric and magnetic field encountered by the particle along its trajectory. In a pure electrostatic ring, $\mathbf{f}$ reduces to

$$
\mathbf{f}=\left(a \gamma-\frac{\gamma}{\gamma^{2}-1}\right) \frac{\mathbf{E} \times \mathbf{v}}{c^{2}},
$$

with $a$ the spin anomaly. At the magic momentum $p c=m c^{2} / \sqrt{a}$ it is exactly $\mathbf{f}=\mathbf{0}$ and the spin remains frozen in its injection (longitudinal) direction.

For the electron it is $m c^{2} \approx 0.51 \mathrm{MeV}$ and $a=(g-2) / 2 \approx 1.159710^{-3}$, so the magic momentum is $p c=15 \mathrm{MeV}$.

\section{Spin Polarimetry for the eEDM}

Spin polarimetry for the pEDM will be performed by nuclear means. This is not practical for 15 $\mathrm{MeV}$ polarized electrons. Among electron spin polarimetry methods, spin polarimetry by undulator synchrotron radiation seems promising [4], [5], [6], [7], [8], since:

1. Radiation will be detected and measured by optical means, using a spectroscope with suitable polarization sensitive detector and probably a lock-in amplifiers. A measurement that should be very "clean" and accurate.

2. Undulator synchrotron radiation shows a line spectrum. Spectral lines from the oscillating electron charge and lines from the oscillating electron magnetic moment have opposite polarization.

3. In a conventional magnetic ring, one would use a magnetic undulator, while in an all-electric bend ring one might want to use an all-electric undulator

\subsection{Radiation from an accelerated charge}

The radiation power from an accelerated charged particle, per solid angle and in the time interval $\Delta t$ is given by the Poynting formula

$$
4 \pi \frac{d W}{d \Omega}=\frac{\mu_{0}}{4 \pi} e^{2} c \int_{\Delta t} A(t)^{2} d t
$$


with $\mathbf{A}^{(q)}$ the vector potential, in a direction defined by $\mathbf{n}$ from the source

$$
\mathbf{A}^{(\mathbf{q})}(\mathbf{t})=\frac{\mathbf{n} \times\left[(\mathbf{n}-\tilde{\beta}) \times \frac{\mathbf{d} \tilde{\beta}}{\mathbf{d t}}\right]}{[\mathbf{1}-\mathbf{n} \cdot \tilde{\beta}]^{2}}
$$

with $\vec{\beta}=\vec{v} / c$ the reduced velocity, and $t$ the retarded time, i.e the time at the point of observation.

\subsection{Radiation from accelerated dipole}

The accelerated particle has a magnetic moment $\vec{\mu}$ proportional to its spin $\mathbf{s}$. In the particle rest frame it is

$$
\vec{\mu}=\frac{g e}{m c} \frac{1}{2} \hbar \mathbf{s}
$$

In the LAB the spin transforms as

$$
\mathbf{S}=\mathbf{s}+\frac{\gamma^{2}}{1+\gamma}(\vec{\beta} \cdot \mathbf{s}) \vec{\beta}
$$

and the accelerated moment produces in the LAB radiation with vector potential [9]

$$
\mathbf{A}^{(\mu)}(\mathbf{t})=\frac{\mathbf{1}}{\mathbf{D}} \frac{\mathbf{d}}{\mathbf{d t}}\left[\frac{\mathbf{n} \times[\mathbf{S}+\mathbf{n} \times(\tilde{\beta} \times \mathbf{S}]}{\mathbf{D}}\right], \mathbf{D}=(\mathbf{1}-\tilde{\beta} \cdot \mathbf{n})
$$

\subsection{Total radiation: charge + moment}

The total radiation vector potential is

$$
\mathbf{A}^{(\mathbf{t})}(\mathbf{t})=\mathbf{A}^{(\mathbf{q})}(\mathbf{t})+\eta \mathbf{A}^{(\mu)}, \eta=\frac{\mathbf{1}}{\mathbf{4}}\left(\frac{\mathbf{g} \gamma}{\mathbf{m c}^{\mathbf{2}}}\right) \hbar \omega .
$$

The contribution from the spin is proportional to $\left(g \gamma / m c^{2}\right)$.

Perform the time derivative in Eq.(7) and obtain, after some work, an expression of $\mathbf{A}^{(\mu)}$ as a sum of one term proportional to $\mathbf{s}$ and one proportional to $\dot{\mathbf{s}}$

$$
\mathbf{A}^{(\mu)}=\mathbf{F}_{1} \mathbf{s}+\mathbf{F}_{\mathbf{2}} \dot{\mathbf{s}}
$$

$F_{1}$ and $F_{2}$ contain both $\vec{\beta}$ and $\dot{\vec{\beta}}$ that will be calculated by the Lorentz equation of motion in an electrostatic undulator (that will not on the spin of the magic particle) and $\mathbf{s}$ and $\dot{\mathbf{s}}$, calculated by the T-BMT equation, respectively.

$$
\text { Lorentz }: \frac{d \mathbf{p}}{d t}=e \mathbf{E}, \mathrm{T}-\mathrm{BMT}: \frac{\mathbf{d s}}{\mathbf{d t}}=\mathbf{f} \times \mathbf{s}
$$

\section{Electrostatic undulator}

Let's pass the electron beam through an electrostatic undulator as schematically shown in Figure 1 (with $\mathrm{N}=2$ periods) Here all components of the spin will remain longitudinal frozen from injection and containing a possible emerging vertical component due to the EDM (the aim of the experiment.) 


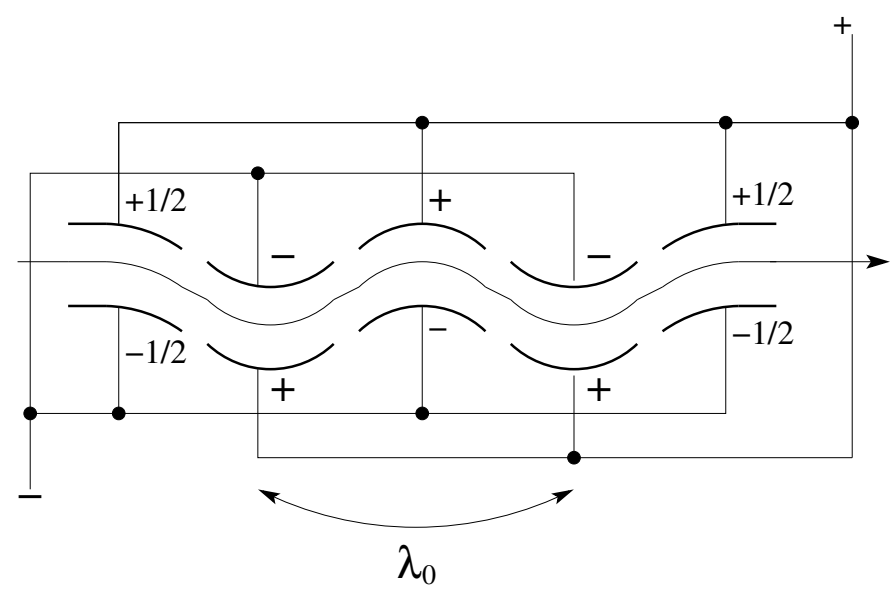

Figure 1. electrostatic undulator

\subsection{Polarized $p, \sigma$ components of $A^{(q)}$ and $A^{(\mu)}$}

In an electric undulator, the equation of motion are

$$
\frac{d \vec{\beta}}{d t}=\vec{\beta} \times \vec{\Omega}, \text { with } \Omega=\frac{e \mathbf{E}}{\beta^{2} c^{2} m \gamma}
$$

In far field (Fraunhofer) the radiation is calculated along $\mathbf{n}$. Components of $\mathbf{A}^{(q)}$ polarized $\|$ to the horizontal plane and $\perp$ to $\mathbf{n}$, and $\mathbf{A}^{(\mu)} \perp$ to $\mathbf{n}$ and to $\mathbf{A}^{(q)}$ are

$$
\mathbf{A}_{p}=\vec{P} \cdot \vec{\Omega} / D^{3}, \quad \mathbf{A}_{\sigma}=\vec{\Sigma} \cdot \vec{\Omega} / D^{3}
$$

with $\vec{P}$ and $\vec{\Sigma}$ some functions.

\subsection{Spectrum of undulator radiation}

The spectrum is obtained by Fourier transform of the vector potential of Eq.(8). In an undulator with $\mathrm{N}$ periods (lines of width $1 / \mathrm{N}$ ) the electric field can be written as

$$
\Omega_{x}=\Omega_{0} \sin \omega_{0} t, \omega_{0}=\frac{2 \pi c}{\lambda_{0}},
$$

with $\lambda_{0}$ the undulator period. The component of the vector potential, spin independent and spin dependent are, respectively, with different polarization and only odd harmonics on the axis

$$
\left\{\begin{array}{l}
A_{p}(t)=\left(1-\gamma^{2} \theta_{b}^{2}\right) \Omega \\
A_{\sigma}(t)=-\left(1+\gamma^{2} \theta_{b}^{2}\right)\left(C_{1} \theta_{b} s_{y}+s_{z}\right) \omega
\end{array},\right.
$$

$\theta_{b}=(k / \gamma) \sin \omega_{0} t$ is the instantaneous angle of the trajectory, with the undulator parameter

$$
k=\frac{e}{2 \pi m_{e} c} \frac{E}{\beta c} \lambda_{0} .
$$

Spin dependent and spin independent components have opposite polarization. Therefore:

the spin state can be measured by looking at the spectral line of correct polarization. 


\section{ICNFP 2013}

\subsection{Competitors}

1. Finite emittance of the beam, that produces a contribution to $A_{\sigma}$ on axis. However this only appears in the even spectral harmonics. The ratio is

$$
\frac{A_{\sigma}^{(s)}}{A_{\sigma}^{(t)}}=\frac{2 \eta}{\gamma} \sqrt{\frac{\beta_{x}}{\epsilon_{x}}}
$$

with $\eta$ defined in Eq.(8). This may show that it is convenient to keep the beam rather wide in $x$.

2. Undulator field Imperfections, since a small $\delta \Omega_{x}$ field residual component with the same periodicity will produce an unwanted $A_{\sigma}$ on axis

3. Chromaticity: electrons in the beam are not all magic, which produces widening of the spectral lines.

\subsection{Wavelength and Intensity of the undulator radiation}

In a transverse electrostatic undulator with: period $\lambda_{0}$, no. of periods $N$ and vertical field $E_{0}$. Angles of the unit vector $\mathbf{n}$ along the radiation are $\theta$, azimuth and $\phi$, latitude.

Wavelength $\lambda$ at harmonic $n$ and photon flux at $n=1$ in a bandwith $\Delta \omega / \omega$ are

$$
\begin{aligned}
& \text { (a) } \lambda_{n}=\lambda_{0} \frac{1+\frac{1}{2} k^{2}+\gamma^{2}\left(\theta^{2}+\phi^{2}\right)}{2 n \gamma^{2}} \\
& \text { (b) } n_{v}=\pi N \alpha \frac{I}{e} \frac{\Delta \omega}{\omega} k^{2}, \alpha \approx \frac{1}{137} \text { (fine struct. constant) }
\end{aligned}
$$

$I$ is the current in the electron beam and $k$ is in Eq.(15). Eq.(a) above shows that the width of the spectral line is $\approx 1 /\left(n \gamma^{2}\right)$. (b) shows that the intensity of the line is proportional to $E^{2}$. 


\subsection{Example - rounded values}

constants

\begin{tabular}{|ll|}
\hline particle & $=$ electron \\
rest mass-energy $m c^{2}[\mathrm{MeV}]$ & $=0.511$ \\
magnetic anomaly $a$ & $=1.1597 \mathrm{e}-3$ \\
fine structure constant $\alpha$ & $=1 / 137=7.299 \mathrm{e}-3$ \\
\hline
\end{tabular}

beam and undulator

\begin{tabular}{|ll|}
\hline ring radius & $=5 \mathrm{~m}$ \\
magic momentum $p c[\mathrm{MeV}]$ & $=15.054$ \\
electric field $E$ & $=750 \mathrm{KV} / \mathrm{m}$ \\
und.period $\lambda_{0}$ & $=10 \mathrm{~cm}$ \\
$\rho$ in the undulator & $=20 \mathrm{~m}$ \\
number of periods $N$ & $=20$ \\
undulator parameter $k$ & $=0.0233$ \\
beam undulation amplitude & $=62 \mu \mathrm{m}$ \\
wavelength $\lambda$ & $=57.92 \mu \mathrm{m}$ \\
$\Delta \omega / \omega$ & $=1 . \mathrm{e}-6$ \\
beam current $I$ & $=1 \mu \mathrm{A}$ \\
photons/sec $n_{v}$ & $\approx 3 \mathrm{e} 12$ \\
\hline
\end{tabular}

\section{References}

[1] A.U.Luccio, paper in this Conference

[2] M. Ternov, AIP Conf Proceedings 343, 35 (1965)

[3] S. A. Belomesthnikh et al., Nucl. Inst. Meth, s227,173, (1984)

[4] D. F. Alferov, Yu. A. Bashmakov and E. G. Bessonov, Sov. Physics-Technical Physics 18, 1336, 1974

[5] A. U. Luccio, Proc. 1993 PAC (IEEE 1993, p. 2175)

[6] A.U.Luccio, BNL-52348, Oct.5, 1992

[7] A.U.Luccio, M.Conte, PAC99, Dubna

[8] A.U.Luccio, and M.Conte SPIN98. Proc 13th Intern. Symposium on High Energy Spin Physics. Protvino, Russia Sept. 8-12, (1998)

[9] J.D.Jackson, Classical Electrodynamics, Wiley, New York, 1962, chapters 11 and 14 